Quim. Nova, Vol. 33, No. 3, 730-733, 2010

\title{
REAPROVEITAMENTO DE ÓXIDOS DE MANGANÊS DE PILHAS DESCARTADAS PARA ELETROCATÁLISE DA REAÇÃO DE REDUÇÃO DE OXIGÊNIO EM MEIO BÁSICO
}

\author{
Daniel C. Rascio, Rodrigo F. B. De Souza, Érico Teixeira Neto, Hugo B. Suffredini e Mauro C. Santos* \\ Centro de Ciências Naturais e Humanas, Universidade Federal do ABC, Rua Santa Adélia 166, 09210-170 Santo André - SP, Brasil \\ Marcelo L. Calegaro \\ Instituto de Química de São Carlos, Universidade de São Paulo, CP 780, 13560-970 São Carlos - SP, Brasil
}

Recebido em 12/3/09; aceito em 6/10/09; publicado na web em 2/3/10

\begin{abstract}
USE OF MANGANESE OXIDES RECOVERED FROM SPENT BATTERIES IN ELECTROCATALYSIS OF OXYGEN REDUCTION REACTION IN ALKALINE MEDIUM. The oxygen reduction reaction was studied in alkaline media using manganese oxides obtained from spent batteries as electrocatalysts. Three processes were used to recover manganese oxides from spent batteries. The particles obtained were in the range from 8 to $11 \mathrm{~nm}$. The electrochemical experiments indicated a good electrocatalytic activity toward oxygen reduction using the different samples and showing approximately a direct transference of 4 electrons during the process. Even though all the processes were efficient, the best result was observed for the prepared sample using reactants of low cost.
\end{abstract}

Keywords: manganese oxide; oxygen reduction; battery metal recovery.

\section{INTRODUÇÃO}

Vários dispositivos que são utilizados no dia a dia, como máquinas térmicas, motores de combustão interna, caldeiras industriais, dentre outras, utilizam combustíveis de origem fóssil como fonte de energia. A utilização deste tipo não renovável de energia produz grandes quantidades de poluentes, destacando-se $\mathrm{CO}_{2}, \mathrm{CO}, \mathrm{NO}_{x}, \mathrm{SO}_{x}$, hidrocarbonetos e materiais particulados, que são responsáveis por boa parte dos problemas ambientais, tais como o efeito estufa e a chuva ácida.

Com o crescente avanço da tecnologia surgiu também nos últimos anos uma grande necessidade por fontes portáteis de energia. No Brasil são produzidas anualmente cerca de 3 bilhões de unidades de pilhas e baterias para suprir esta necessidade. ${ }^{1}$ Segundo dados da ABINEE (Associação Brasileira da Indústria Eletroeletrônica) a porcentagem de pilhas alcalinas vendidas no Brasil representa cerca de $30 \%$ do total das vendas no mercado. Em contrapartida, as pilhas alcalinas dominam o mercado nos EUA por durarem cerca de seis vezes mais que as pilhas de zinco-carvão. ${ }^{1}$

As pilhas citadas acima após seu esgotamento energético são descartadas, tendo por destinos finais locais inadequados. Isto causa grandes impactos ambientais, principalmente relacionados à poluição de lençóis freáticos pela contaminação de metais pesados como o manganês, reconhecidamente um dos principais componentes deste tipo de pilha. ${ }^{2}$

Este metal, presente na forma de óxidos nas pilhas, pode ser recuperado e empregado no uso e desenvolvimento de células a combustível como eletrocatalisador para a reação de redução do oxigênio. Esta reação impõe grandes limitações para o desenvolvimento de células a combustível, ${ }^{3}$ que são uma boa opção na substituição das atuais fontes de energia, podendo satisfazer tanto as necessidades energéticas supridas pelos combustíveis fósseis como para aplicação em dispositivos portáteis que utilizam pilhas e baterias.

A reação de redução de oxigênio (RRO) é uma reação multieletrônica que inclui algumas etapas elementares em seu mecanismo. Esta transformação química ocorre segundo dois mecanismos globais bastante discutidos na literatura: o mecanismo direto (4 elétrons, Figura 1 - I) e, o mecanismo de formação do peróxido (2 elétrons)., ${ }^{4,5}$ A distinção entre os dois mecanismos torna-se difícil pelo fato da

\footnotetext{
*e-mail: mauro.santos@ufabc.edu.br
}

redução direta também poder envolver a formação de peróxido, desde que este permaneça adsorvido na superfície do eletrodo sem sofrer dessorção ou decomposição, e a sua redução ocorra subsequentemente. Este processo também envolve 4 elétrons, porém é chamado de mecanismo em série (Figura 1 - II). Em potenciais mais negativos que o mecanismo via 4 elétrons ocorre o mecanismo via 2 elétrons, sendo este conhecido por mecanismo paralelo (Figura 1 - III).

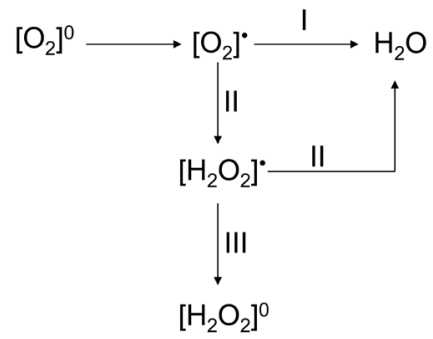

Figura 1. Esquema do mecanismo de reação de redução do oxigênio

Sabe-se que os melhores eletrocatalisadores para a reação de redução do oxigênio (que reduzem as moléculas de $\mathrm{O}_{2}$ pela via direta de 4 elétrons) contêm metais nobres em sua composição, como Pd e Pt. Entretanto, estes metais são raros e caros, o que limita o avanço do uso em células a combustível. Células a combustível que utilizam eletrólito alcalino são muito estudadas, isto ocorre devido à possibilidade da utilização de metais não nobres para compor tanto os materiais de cátodo quanto de ânodo. ${ }^{6-8}$

Os fatos discutidos incentivam a busca por novos materiais que sejam capazes de substituir os metais nobres e diminuir o custo final das células a combustível. Muitos estudos já foram realizados visando o desenvolvimento de eletrocatalisadores eficientes de baixo custo para aplicações em meio básico, como células a combustível alcalinas e baterias metal/ar. ${ }^{9}$

Assim, visando a otimização da reação de redução de oxigênio para células a combustível, a redução dos custos de produção dos eletrocatalisadores para esta reação e dos impactos ambientais causados pelo $\mathrm{Mn}$ proveniente do descarte inadequado de pilhas, no presente trabalho estudou-se a RRO utilizando-se óxidos de Mn recuperados de pilhas por diferentes métodos de obtenção. 


\section{PARTE EXPERIMENTAL}

\section{Obtenção de óxidos de manganês extraídos de pilhas secas}

Os óxidos de Mn foram extraídos de pilhas comuns usadas, que foram desmontadas, retirando-se seus invólucros externos de aço para se ter acesso ao invólucro interno constituído de Zn. Este invólucro interno foi aberto para se ter acesso à pasta eletrolítica onde estão presentes os óxidos de manganês que foram recolhidos.

Esse material foi, então, lavado abundantemente com água Mili$\mathrm{Q}^{\circledR}$ e dividido em 4 alíquotas, sendo 3 delas destinadas a diferentes métodos de tratamento (amostras A, B e C) e outra (amostra D) guardada para servir de referência na caracterização eletroquímica.

Foram escolhidos três métodos para o tratamento do material retirado da pilha, sendo que dois deles envolveram a neutralização da alíquota de interesse e posterior tentativa de isolamento do $\mathrm{MnO}_{2}$ mediante a adição de $\mathrm{H}_{2} \mathrm{O}_{2}$. A diferença entre eles é o emprego de reagentes de padrão analítico ou comercial. O terceiro método envolve uma fusão ácida com $\mathrm{KHSO}_{4}$ que se mostrou eficiente na extração de óxidos de Mn de pilhas de Li-Mn, segundo Paulino et al.. ${ }^{10}$

O material chamado "Amostra A" foi misturado a uma solução de $\mathrm{NaOH} 5 \mathrm{~mol} \mathrm{~L}^{-1}$ (Synth) sob constante agitação por 30 min.. Em seguida, foi adicionado $5 \%$ do volume da solução de peróxido de hidrogênio 20 volumes (Synth). Após mais 30 min de agitação constante, a solução foi filtrada e o pó resultante foi lavado abundantemente com água do sistema Milli-Q até reduzir o $\mathrm{pH}$ para próximo de 7 e colocado em estufa para secagem completa em uma temperatura de $60^{\circ} \mathrm{C}$ por $2 \mathrm{~h}$.

A “Amostra B" recebeu o mesmo tratamento da amostra anterior, diferenciando-se pelo fato de que o objetivo foi o de diminuir custos de obtenção. Neste caso, reagentes comuns foram utilizados. No lugar do hidróxido de sódio P.A., foi utilizada soda cáustica destinada à limpeza, marca Gaviões. Para substituir o peróxido de hidrogênio P.A., foi utilizada água oxigenada 40 volumes ADV Beauty.

Finalmente, a "Amostra C" foi submetida a uma reação de fusão com $\mathrm{KHSO}_{4}$ (Merck), conforme descrito por Paulino et al. ${ }^{10}$ À solução resultante deste processo (de tonalidade rósea, devido à presença de íons de $\mathrm{Mn}^{2+}$ ) foi adicionado pó de carbono Vulcan XC-72, solução de hidróxido de sódio (P.A.) $5 \mathrm{~mol} \mathrm{~L}^{-1}$ e $5 \%$ de peróxido de hidrogênio (grau analítico) seguindo-se o mesmo procedimento usado para a amostra A.

\section{Caracterização física}

Os materiais obtidos foram caracterizados fisicamente pelas técnicas espectroscópicas de energia dispersiva (EDS) utilizando-se um microscópio eletrônico de varredura (JEOL JSM-5900LV SEM com 30 kV) e difração de raios-X (DRX) com um difratômetro Rigaku modelo Miniflex, utilizando-se radiação $\mathrm{Cu}-\mathrm{K} \alpha(1,541 \AA, 30 \mathrm{kV}$ e $15 \mathrm{~mA})$ para avaliar as fases presentes nos eletrocatalisadores e tamanho médio de cristalito.

\section{Caracterização eletroquímica}

Os experimentos eletroquímicos foram realizados em um potenciostato/galvanostato Autolab PGSTAT 302 N. Foi utilizado como eletrodo de trabalho um eletrodo rotatório com disco de carbono vítreo $\left(0,2 \mathrm{~cm}^{2}\right)$ e anel de Pt para detecção de peróxido de hidrogênio, um eletrodo de Pt com área de $1 \mathrm{~cm}^{2}$ como contraeletrodo e um eletrodo de $\mathrm{Ag} / \mathrm{AgCl}$ saturado como referência.

O eletrocatalisador foi depositado sobre o disco de carbono vítreo do eletrodo de trabalho na forma de camada ultrafina porosa. Para isso, foram misturadas $8 \mathrm{mg}$ do eletrocatalisador com $2 \mathrm{~mL}$ de água Milli-Q, agitando-se o conjunto em um banho ultrassônico por 5 min. Em seguida, foram acrescentados $20 \mu \mathrm{L}$ de uma solução de Náfion ${ }^{\circledR}$ 5\%, homogeneizando-se em banho ultrassônico por 20 min. Foram depositados $20 \mu \mathrm{L}$ desta suspensão de eletrocatalisador sobre a superfície do carbono vítreo e o eletrodo foi acondicionado em estufa a $60{ }^{\circ} \mathrm{C}$ por $15 \mathrm{~min}$ para secagem.

Os experimentos eletroquímicos foram realizados utilizando-se uma solução $1 \mathrm{~mol} \mathrm{~L}^{-1}$ de $\mathrm{NaOH}$ supra-puro. O eletrólito foi purgado com $\mathrm{N}_{2}$ ultrasseco por 15 min antes dos experimentos voltamétricos e por 15 min com $\mathrm{O}_{2}$ grau zero para saturar a solução nos experimentos de redução de oxigênio com curvas de polarização em estado estacionário.

Para servir de padrão comparativo de desempenho dos eletrocatalisadores para a reação de redução eletroquímica de oxigênio, foi utilizado um eletrocatalisador de $\mathrm{Pt} / \mathrm{C} 20 \% \mathrm{~m} / \mathrm{m}$ produzido pelo método dos precursores poliméricos, adaptado para aplicação em carbono de alta área como substrato. ${ }^{11-13}$

\section{RESULTADOS E DISCUSSÃO}

\section{Caracterização física}

A composição dos materiais foi determinada por espectroscopia de energia dispersiva (EDS) conforme apresentado na Tabela 1.

Tabela 1. EDX para as amostras tratadas pelos diferentes métodos: A (material retirado da pilha e tratado com $\mathrm{NaOH}$ p.a. e $\mathrm{H}_{2} \mathrm{O}_{2}$ p.a.), $\mathrm{B}$ (material retirado da pilha e tratado com $\mathrm{NaOH}$ e $\mathrm{H}_{2} \mathrm{O}_{2}$ de baixo custo), $\mathrm{C}$ (material retirado da pilha e realizado tratamento conforme ref. 10

\begin{tabular}{lccc}
\hline Metal $\backslash$ Catalisador & $\mathrm{A}$ & $\mathrm{B}$ & $\mathrm{C}$ \\
\hline $\mathrm{Al}$ & $4,05 \%$ & $2,2 \%$ & $5,2 \%$ \\
$\mathrm{Ca}$ & - & - & $0,5 \%$ \\
$\mathrm{Mn}$ & $90,43 \%$ & $93,4 \%$ & $90,6 \%$ \\
$\mathrm{Si}$ & $2,66 \%$ & $1,75 \%$ & - \\
$\mathrm{Zn}$ & $2,85 \%$ & $2,12 \%$ & $2,72 \%$ \\
\hline
\end{tabular}

Observam-se altos percentuais (>90\%) de compostos de Mn em todas as amostras, comprovando a eficácia dos métodos empregados na extração do $\mathrm{Mn}$ das pilhas. As amostras A, B e C apresentam quantidades entre 2 e $5 \%$ de $\mathrm{Al}$ e 2 a $2,85 \%$ de $\mathrm{Zn}$, que são provenientes da pilha, uma vez que um dos eletrodos é constituído de zinco. A presença de $\mathrm{Al}$ e Si pode ser advinda de impurezas presentes na pasta eletrolítica da pilha, as porcentagens destes elementos parecem elevadas devido à análise de EDS realizada ignorar o carbono presente.

Na Figura 2 são apresentados os difratogramas de raios-X das amostras. Todos os materiais apresentaram uma mistura de diferentes óxidos de Mn em sua composição, com alguns picos localizados em $2 \theta=28,8^{\circ}, 37,4^{\circ}$ e $56,8^{\circ}$ característicos de $\mathrm{MnO}_{2} .^{14,15}$ Os padrões obtidos também são típicos de $\mathrm{Mn}_{3} \mathrm{O}_{4} / \mathrm{C}$ com pequenas contribuições de $\mathrm{MnO}$ e $\mathrm{MnO}_{2} \cdot{ }^{16}$

Um fator importante a ser observado é que os diferentes métodos de preparação empregados produziram os mesmos tipos de óxidos, variando apenas a predominância de uma fase em relação à outra. A amostra A apresentou predominantemente $\mathrm{MnO}_{2}$, enquanto que as amostras $\mathrm{B}$ e C apresentaram maior quantidade de $\mathrm{Mn}_{3} \mathrm{O}_{4}$ em sua composição.

Os dados obtidos por DRX foram analisados utilizando-se a equação de Sherrer para a determinação do tamanho médio de cristalito dos eletrocatalisadores, conforme descrito por Lima et al., ${ }^{17} \mathrm{e}$ os resultados obtidos são apresentados na Tabela 2.

Tabela 2. Tamanhos médios de cristalito das amostras

\begin{tabular}{lc}
\hline & Tamanho médio / nm \\
\hline Amostra A & 8 \\
Amostra B & 11 \\
Amostra C & 10 \\
\hline
\end{tabular}




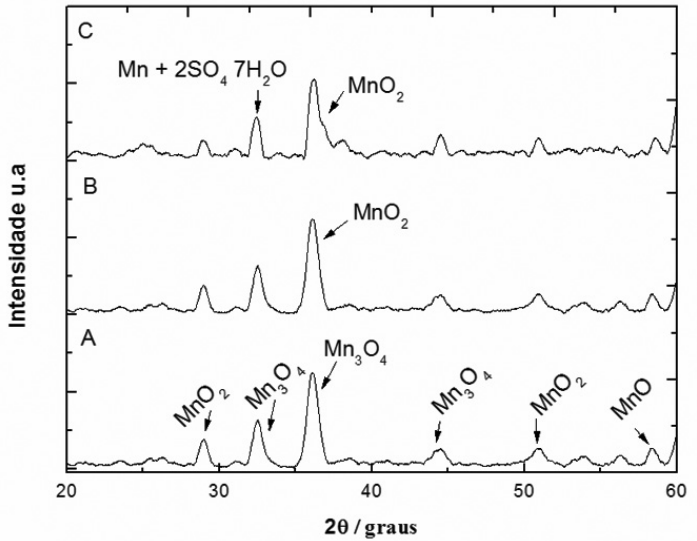

Figura 2. DRX das amostras: (1) Amostra A, (2) Amostra B e (3) Amostra C

Os valores obtidos são característicos de nanopartículas de óxidos de Mn conforme apresentado em trabalhos anteriores, ${ }^{17,18}$ onde se concluiu que o tamanho médio de cristalito pode influenciar diretamente as propriedades eletrocatalíticas do material.

\section{Caracterização eletroquímica}

Os experimentos eletroquímicos realizados por meio de voltamogramas cíclicos são apresentados na Figura 3. Os perfis voltamétricos observados são característicos de uma mistura de óxidos de Mn e são muito similares àqueles encontrados na literatura. ${ }^{19-21}$

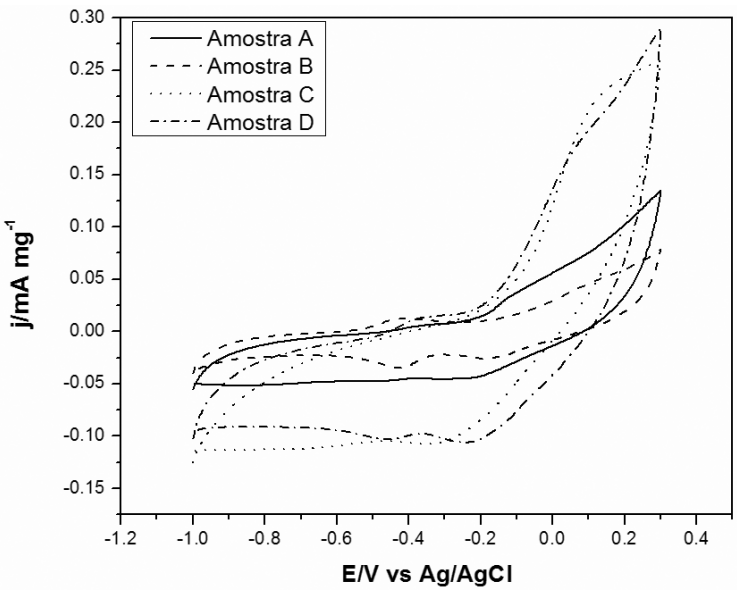

Figura 3. Perfis voltamétricos obtidos para eletrocatalisadores de $\mathrm{Mn} / \mathrm{C}$ recuperados de pilhas usadas, em meio de $\mathrm{NaOH} 1 \mathrm{Mol} \mathrm{L}^{-1}$. Velocidade de varredura de potenciais $=10 \mathrm{mV} \mathrm{s}^{-1}, E_{i}-1 \mathrm{Ve} E_{f} 0,25 \mathrm{~V}(\mathrm{vs} . \mathrm{Ag} / \mathrm{AgCl})$

Comparando-se as curvas voltamétricas para os eletrocatalisadores constituídos de óxidos de manganês com as obtidas por Lima et al.,${ }^{17}$ observa-se que os perfis são típicos de uma mistura de diferentes óxidos de Mn. Esses perfis também foram observados por outros autores ${ }^{22,23}$ em condições experimentais similares às deste trabalho.

Os picos voltamétricos na Figura 3 estão associados a uma série de processos de oxi-redução onde a reação principal é a redução e reoxidação do $\mathrm{MnO}_{2}$ e $\mathrm{Mn}_{2} \mathrm{O}_{3}$ ( $\mathrm{MnIV} / \mathrm{MnIII}$ e $\left.\mathrm{MnIII} / \mathrm{MnII}\right)$, de acordo com as reações 1 e $2:^{24}$

$\mathrm{MnO}_{2}+\mathrm{H}_{2} \mathrm{O}+\mathrm{e}^{-} \rightarrow \mathrm{MnOOH}+\mathrm{OH}^{-}$

$\mathrm{MnOOH}+\mathrm{H}_{2} \mathrm{O}+\mathrm{e}^{-} \rightarrow \mathrm{Mn}^{2+}+3 \mathrm{OH}^{-}$
A reação 2 pode ser considerada completamente inibida, devido à baixa condutividade do $\mathrm{MnOOH}$. A reação de redução do $\mathrm{Mn}_{2} \mathrm{O}_{3}$ ocorre em uma única etapa, de acordo com a reação 3 , e sua oxidação leva à formação de $\mathrm{MnO}_{2}$, de acordo com a reação $4::^{23}$

$$
\begin{aligned}
& \mathrm{Mn}_{2} \mathrm{O}_{3}+3 \mathrm{H}_{2} \mathrm{O}+2 \mathrm{e}^{-} \rightarrow 2 \mathrm{Mn}(\mathrm{OH})_{2}+2 \mathrm{OH}^{-} \\
& \mathrm{Mn}_{2} \mathrm{O}_{3}+2 \mathrm{OH}^{-} \rightarrow 2 \mathrm{MnO}_{2}+\mathrm{H}_{2} \mathrm{O}+2 \mathrm{e}^{-}
\end{aligned}
$$

O desempenho dos eletrocatalisadores para a RRO é apresentado na Figura 4, em um gráfico que mostra as curvas de polarização em estado estacionário $(\omega=1600$ RPM). Em (a) são apresentados os dados do anel e em (b) encontram-se as medidas do disco. Os potenciais de redução de $\mathrm{O}_{2}$ para cada amostra são apresentados na Tabela 3.

Tabela 3. Potenciais de redução de $\mathrm{O}_{2}$ para $\mathrm{Mn} / \mathrm{C}$ recuperado de pilhas

\begin{tabular}{lc}
\hline & Potencial de redução do oxigênio \\
\hline Amostra A & $-0,13 \mathrm{~V}$ \\
Amostra B & $-0,12 \mathrm{~V}$ \\
Amostra C & $-0,15 \mathrm{~V}$ \\
Amostra D & $-0,12 \mathrm{~V}$ \\
\hline
\end{tabular}

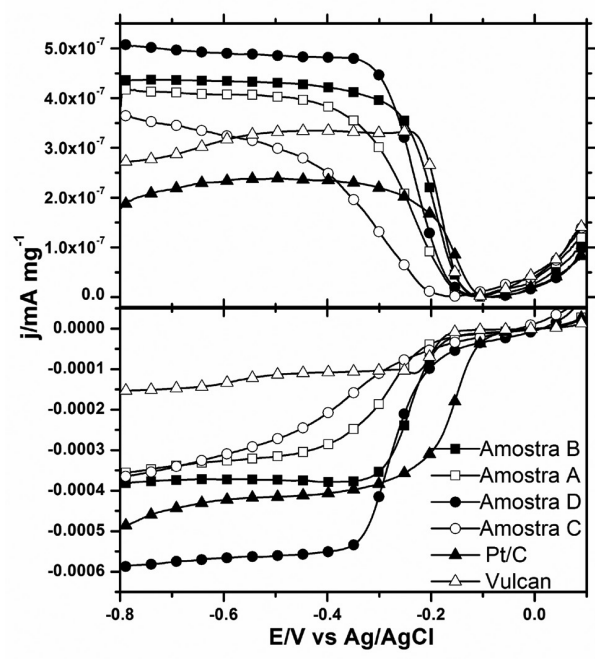

Figura 4. Curvas de polarização em estado estacionário para os diferentes eletrocatalisadores de $\mathrm{Mn} / \mathrm{C}$ recuperados de pilhas usadas, em meio de $\mathrm{NaOH}$ $1 \mathrm{Mol} \mathrm{L}^{-1}$ : (a) corrente do anel e (b) corrente no disco. $\Phi=1600 \mathrm{rpm}$. A curva de Pt/C foi inserida para comparação. Potencial do anel $=0,15 \mathrm{~V}$

Na Tabela 3 observa-se que houve grande proximidade entre os potenciais de início para a redução do oxigênio obtidos com as amostras D, A e B e uma ligeira discrepância da amostra C (em torno de 0,025 V). Com as amostras D e B foi observado o mesmo potencial para a redução de oxigênio. A amostra $B$, porém, apresentou menor densidade de corrente no anel, evidenciando-se menor produção de peróxido de hidrogênio (um dos indícios de que a reação seguiu pelo mecanismo direto).

A fim de tornar a análise quantitativa, um gráfico de KouteckyLevich $\left(\mathrm{j}^{-1}\right.$ vs $\left.\varpi^{-1 / 2}\right)$ foi construído com dados das curvas de polarização em diferentes velocidades de rotação do eletrodo. O número de elétrons envolvidos na RRO pode ser estimado a partir do coeficiente angular deste gráfico, ${ }^{25}$ de acordo com a Equação 5.

$$
1 /{ }_{\mathrm{j}}=1 / j_{\mathrm{k}}+1 / j_{\mathrm{dl}}=1 /\left(\mathrm{nFkC}_{\mathrm{O} 2}^{\mathrm{b}}\right)-1 /\left(0.62 \mathrm{nFD}^{3 / 2}{ }_{\mathrm{O} 2} v^{-1 / 6} \mathrm{C}_{\mathrm{O} 2}^{\mathrm{b}} \omega^{1 / 2}\right)
$$

onde $\mathrm{J}_{\mathrm{k}}$ e $\mathrm{J}_{\mathrm{dl}}$ são as correntes cinéticas e de limite difusional; $\mathrm{F}$ é a constante de Faraday; k é a constante de transferência do primeiro 
elétron no mecanismo de redução; $\mathrm{D}_{\mathrm{O} 2}$ é o coeficiente de difusão do $\mathrm{O}_{2}$; $\varpi$ é a velocidade de rotação do eletrodo; $v$ é a viscosidade cinemática da solução e $\mathrm{C}_{\mathrm{O} 2}$ é a concentração de $\mathrm{O}_{2}$ no seio da solução.

Em um diagrama de Koutecky-Levich, a inclinação das retas apresenta relação direta com o número de elétrons (n) envolvidos na $\mathrm{RRO}$, de acordo com a Equação 5. Assim, assume-se $\mathrm{n}=2$ para o carbono Vulcan e $n=4$ para $\mathrm{Pt} / \mathrm{C}$, uma vez que estes são os eletrocatalisadores que apresentam baixa e alta eficiências neste processo, respectivamente. Os pontos são determinados a partir das curvas de polarização em um determinado potencial, onde o controle da reação é regido pela corrente cinética. Desta maneira, é possível fazer uma estimativa do número de elétrons envolvidos na reação.

O diagrama de Koutecky-Levich obtido para os diferentes eletrocatalisadores é apresentado na Figura 5.

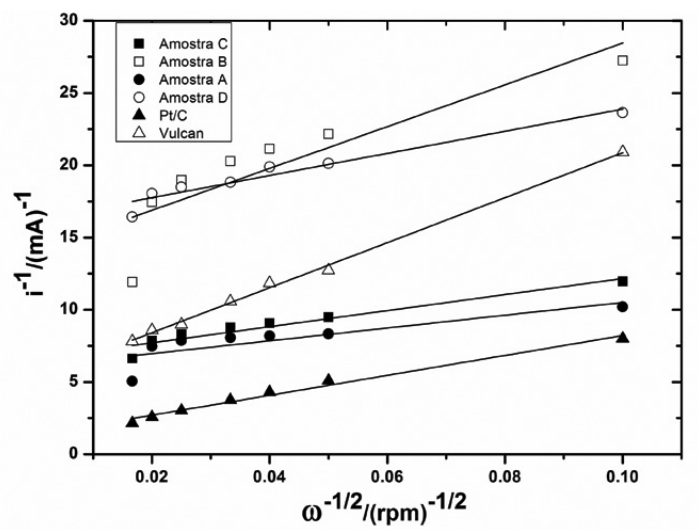

Figura 5. Diagrama de Koutecky-Levich para os eletrocatalisadores de Mn/C recuperados de pilhas usadas, em -0,22 $\mathrm{V}$ e solução de $\mathrm{NaOH} 1 \mathrm{Mol} \mathrm{L}^{-1}$. Os valores de Pt/C e carbono Vulcan foram inseridos para comparação

Neste gráfico é possível observar grande semelhança na inclinação das retas correspondentes às amostras $\mathrm{B}, \mathrm{C}$ e D, que se assemelham com a inclinação da reta de $\mathrm{Pt} / \mathrm{C}$, obtendo-se assim um número de elétrons transferidos relativamente próximo a 4. Esta é uma evidência de que estes eletrocatalisadores foram capazes de reduzir o oxigênio pelo mecanismo direto ou em série, onde o produto final é a água. A reta correspondente à amostra $\mathrm{A}$ apresenta inclinação próxima da reta correspondente ao carbono Vulcan (onde $n=2$ ), indicando que este eletrocatalisador reduziu o oxigênio pelo mecanismo paralelo, sendo que o produto final é o peróxido. O bom desempenho do material da amostra B, evidenciado pelo alto potencial para a redução do oxigênio (com baixas correntes obtidas no anel) e um número de transferência de elétrons próximo a 4 pode ser explicado pelo fato de que o tratamento ao qual foi submetida a amostra permitiu a formação de grande quantidade de $\mathrm{MnO}_{2}$, que é o óxido de $\mathrm{Mn}$ que apresenta maior atividade eletrocatalítica para a reação de redução do oxigênio. ${ }^{15}$

\section{CONCLUSÕES}

Os eletrocatalisadores para reação de redução de oxigênio, produzidos com óxidos de manganês extraídos de pilhas descartadas, foram estudados em meio alcalino e se mostraram eficientes para tal fim, principalmente os produzidos com reagentes de baixo custo. Os três processos empregados para a recuperação destes óxidos mostraram-se eficientes e apresentaram grande quantidade de óxidos na composição final das amostras, como observado nas análises de EDS e DRX com tamanhos de partícula na ordem de 8 a $11 \mathrm{~nm}$. Os experimentos eletroquímicos com as amostras comprovaram uma boa atividade eletrocatalítica para a reação de redução de oxigênio, com número de elétrons transferidos próximo ao obtido com $\mathrm{Pt} / \mathrm{C}$ (transferência de 4 elétrons durante o processo - redução direta), sendo que o melhor resultado observado foi para a amostra tratada com reagentes de baixo custo, por apresentar uma predominância da fase $\mathrm{MnO}_{2}$ já mostrado na literatura como efetivo para a RRO. Além da eficiência do catalisador em si, é possível apontar outros pontos positivos desses materiais, como o custo ambiental, pois se retira do ambiente um resíduo tóxico e de difícil destinação; e o custo, pois no catalisador de melhor desempenho utilizaram-se materiais de baixo custo como soda caústica comercial e água oxigenada, ao invés de reagentes de grau analítico, mostrando que a pureza do material não beneficia necessariamente o processo.

\section{AGRADECIMENTOS}

À FAPESP (Processos №s 05/59992-6, 07/05155-1 e 08/58789$0)$, CNPq, CAPES e UFABC. E. Teixeira Neto agradece ao LMELNLS pelo uso do microscópio JEOL JSM-5900 LV. À Profa. V. Constantino, do IQ-USP pela análise de difração de raios-X.

\section{REFERÊNCIAS}

1. http://abinee.org.br, acessada em Dezembro 2008.

2. Agourakis, D. C.; Camargo, I. M. C.; Cotrim, M. B.; Flues, M.; Quim. Nova 2006, 29, 960.

3. Srinivasan, S.; J. Electrochem. Soc. 1989, 41C, 136.

4. Yeager, E. B.; Electrochim. Acta 1984, 29, 1527.

5. Yeager, E. B.; J. Mol. Catal. 1986, 38, 5.

6. Vogel, W.; Lundquist, J. T.; J. Electrochem. Soc. 1970, 117, 886.

7. Kiros, Y.; Schwartz, S.; J. Power Sources 1991, 36, 547.

8. Kiros, Y.; Lindstron, O.; Kaimakis, T.; J. Power Sources 1993, 45, 219.

9. Chakkaravarth, C.; Abdul Waheed, A. K.; Udupa, H. V. K.; J. Power Sources 1981, 6, 203.

10. Paulino, J. F.; Busnardo, N. G.; Afonso, J. C.; Quim. Nova 2007, 30, 718.

11. Freitas, R. G.; Santos, M. C.; Oliveira, R. T. S.; Bulhões, L. O. S.; Pereira, E. C.; J. Power Sources 2006, 158, 164.

12. Freitas, R. G.; Marchesi, L. F.; Oliveira, R. T. S.; Mattos-Costa, F. I.; Pereira, E. C.; Bulhões, L. O. S.; Santos, M. C.; J. Power Sources 2007, $171,373$.

13. De Souza, R. F. B.; Flausino, A. E. A.; Rascio, D. C.; Oliveira, R. T. S.; Neto, E. T; Calegaro, M. L; Santos, M. C.; Appl. Catal. B 2009, 91, 516.

14. JCPDS - Joint Committee on Powder Difraction Standards, Pennsylvania, 1980.

15. Lima, F. H. B.; Calegaro, M. L.; Ticianelli, E. A.; Electrochim. Acta 2007, 52, 3732 .

16. Lamminen, J.; Kivisaari, J.; Lampinen, J.; Viitanen, M.; Vuorisalo, J.; J. Electrochem. Soc. 1991, 138, 905.

17. Lima, F. H. B.; Calegaro, M. L.; Ticianelli, E. A.; J. Electroanal. Chem. 2006, 590, 152.

18. Calegaro, M. L.; Lima, F. H. B.; Ticianelli, E. A.; J. Power Sources 2006, 158, 735 .

19. Bezdicka, P.; Grygar, T.; Klapste, B.; Vondrak, J.; Electrochim. Acta 1999, 45, 913

20. McBreen, J.; Electrochim. Acta 1975, 20, 221.

21. Matsuki, K.; Kamada, H.; Electrochim. Acta 1986, 31,13.

22. Brenet, J. P.; J. Power Sources 1979, 4, 183.

23. Klapste, B.; Vondrak, J.; Velicka, J.; Electrochim. Acta 2002, 47, 2365.

24. Lima, F. H. B.; Calegaro, M. L.; Ticianelli, E. A.; Russian, J.; Electrochemistry 2006, 42, 12.

25. Mao, L.; Zhang, D.; Sotomura, T.; Nakatsu, K.; Koshiba, N.; Ohaka, T.; Electrochim. Acta 2003, 48, 1015. 